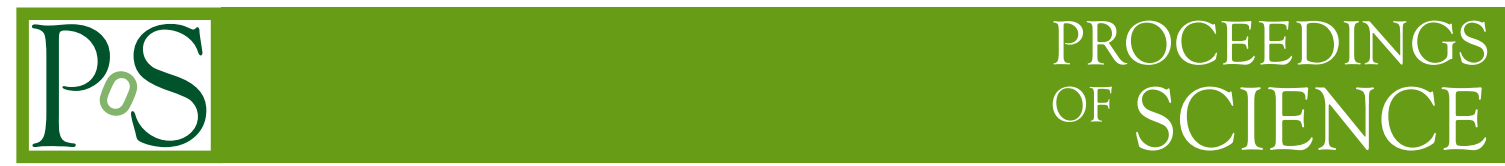

\title{
Emittance Exchange in MICE
}

\section{Bonesini}

Sezione INFN Milano Bicocca,

Dipartimento di Fisica G. Occhialini, Universitá di Milano Bicocca, Milano, Italy

(on behalf of the MICE Collaboration)

For a muon collider a 6D cooling is necessary. Emittance exchange using wedge absorbers may be employed for the required longitudinal ionization cooling. A polyethylene wedge has been manufactured and placed in MICE for such a scope. Data have been collected for both direct emittance exchange (longitudinal emittance decreases) and reverse emittance exchange (transverse emittance decreases). Simulation has demonstrated the feasibility of the method, while data analysis is in progress.

European Physical Society Conference on High Energy Physics - EPS-HEP2019 -

10-17 July, 2019

Ghent, Belgium 


\section{Introduction}

When muons are produced by proton collision with a target, they occupy a large phase-space volume. To meet the acceptance requirements of a storage ring, these muons must be cooled. Ionization cooling [1] is the only viable process to cool the muon beam to the required phase-space density on a timescale smaller than the muon lifetime $(\tau \sim 2.6 \mu \mathrm{s})$. As shown in the right panel of figure 1 for a muon collider both reduction in transverse emittance and in longitudinal emittance are needed. While the transverse cooling of a muon beam may be obtained via "ionization cooling", a direct longitudinal cooling is not feasible, due to the energy-loss straggling that increases the energy spread. The way out is to transfer a fraction of the cooling effect from transverse to longitudinal phase space ("emittance exchange"), as shown schematically in the left panel of figure 1. Clearly this is at the expense of a reduced transverse cooling. In emittance exchange a muon beam is passed through a dipole magnet to create both a position spread and a position-energy correlation in the beam. The beam is passed then through a wedge absorber of specific thickness to eliminate the momentum dispersion. The beam has thus an increased longitudinal phase-space density and a reduced transverse phase-space density. In this way, the emittance has been exchanged. The transverse emittance can then be reduced by ionisation cooling resulting in an overall reduction in $6 \mathrm{D}$ emittance. In reverse emittance exchange the beam is first passed through a wedge and then through a magnetic dipole. This allows one to increase the transverse phase-space density at the expense of decreased longitudinal phase-space density. Thus the emittance exchange has been reversed.
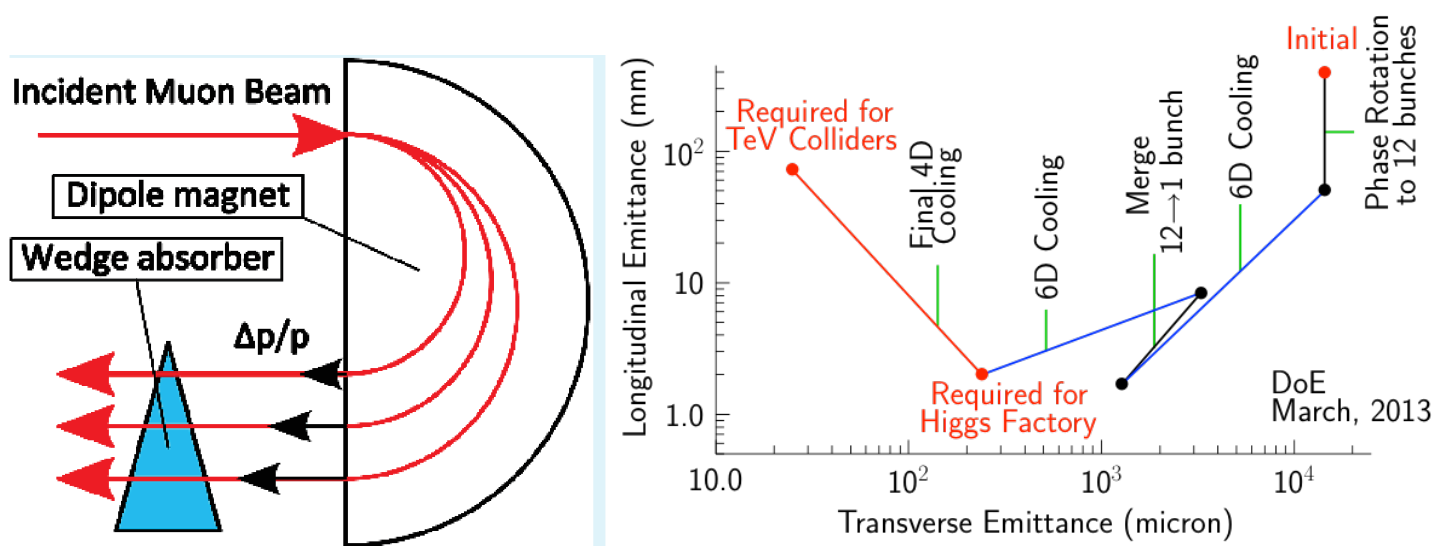

Figure 1: Left: principle of emittance exchange to get $6 \mathrm{D}$ cooling. Dispersion is used to create an appropriate correlation between momentum and transverse position/path length [courtesy of Muons Inc]. Right: cooling path in emittance space for a $\mu^{+} \mu^{-}$collider from reference [2].

\section{The MICE ionization cooling experiment}

In the MICE experiment at RAL [3], a titanium target is dipped into a circulating proton beam from ISIS creating pions, which are subsequently gathered in a quadrupole magnet. These pions then decay to muons in the transport line. The MICE beamline is fully described in references [4], 
[5]. The muon beam is passed through a series of detectors in the cooling channel. The Time-ofFlight stations [6] ( 60 ps resolution), a Kloe-Light calorimeter and an Electron-Muon Ranger [7] are used to distinguish muons from electrons and pions, while the two tracking detectors [8] either side of the absorber, immersed in a uniform multi-Tesla magnetic field, measure the position and momentum of each particle. This is done via five stations of scintillating fibres.
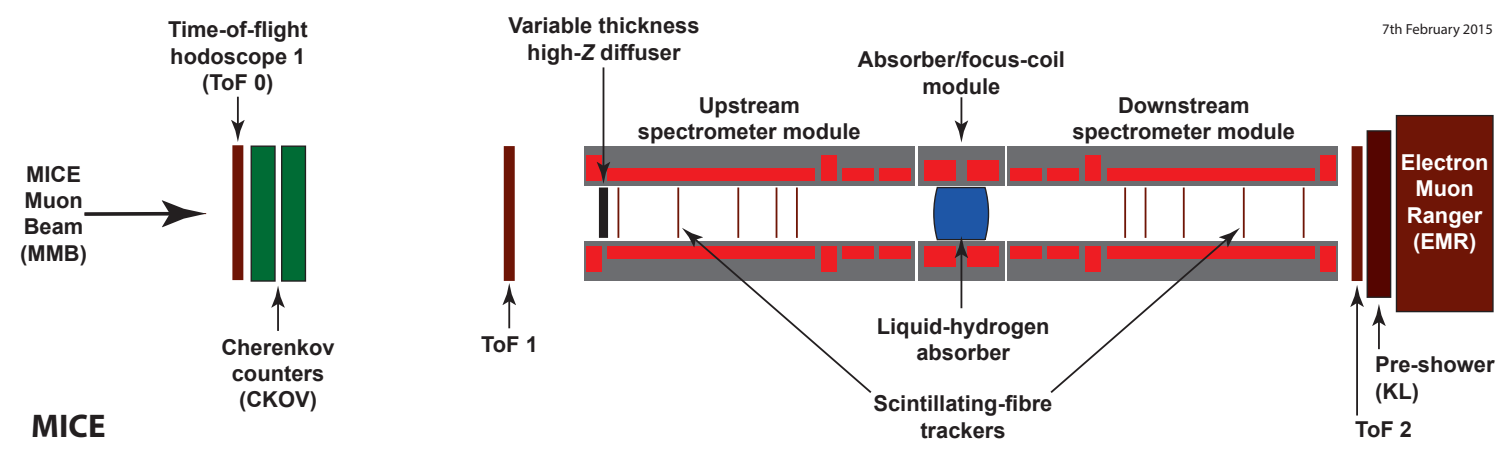

Figure 2: Layout of the MICE experiment at RAL. The cooling channel is put between two magnetic spectrometers and two TOF stations to measure particle parameters. Beam is impinging from the left.

Position and momentum measurements made in each tracker allow the comparison of the phase-space density before and after the absorber. MICE has used lithium hydride and liquid hydrogen as absorbers which have shown an increase in the transverse phase-space density after the muon beam has passed through. Emittance measurements are performed on a single particle basis by measuring $x, y, p_{x}, p_{y}, E, t$ for each muon. In this way an unprecedented precision is obtained [9]. Besides the previous flat absorbers, a 45-degrees opening angle polyethylene wedge has been used to study emittance exchange as shown in figure 1.

Unfortunately the initial ambitious goal of the MICE experiment [3], [10]: the study of a fully engineered cooling cell of the proposed US Study 2 [11] had to be downscaled to a demonstration of ionization cooling [12] and a full study of its parameters for lack of funding.

\section{How to see the effect}

A 45-degrees polyethylene wedge has been manufactured and installed in the focus coil module of MICE. About 14 million triggers were collected with the wedge absorber in situ in 2017. The MICE muon beam has a small natural dispersion, at the absorber location, which is not enough to demonstrate emittance exchange. To demonstrate emittance exchange in MICE a beam selection procedure must be applied where a dispersive beam is selected and then tracked through the wedge and the MICE cooling channel. As MICE uses a particle by particle measurement system, a muon beam may be constructed by assembling individual measurements. A dispersive beam can then be selected from this assembled beam by applying a weighting procedure [13]. Figure 3 shows an example, where the figure in the left panel, a two-dimensional multivariate Gaussian, with normalized emittance $\varepsilon_{x}=15 \mathrm{~mm}, \beta_{x}=334 \mathrm{~mm}$ and $\alpha_{x}=0$ has been moment reweighted by a particular choice of weights. The result is a beam with normalized emittance $\varepsilon_{x}=4.2 \mathrm{~mm}, \beta_{x}=260 \mathrm{~mm}$ and $\alpha_{x}=-0.75$. 

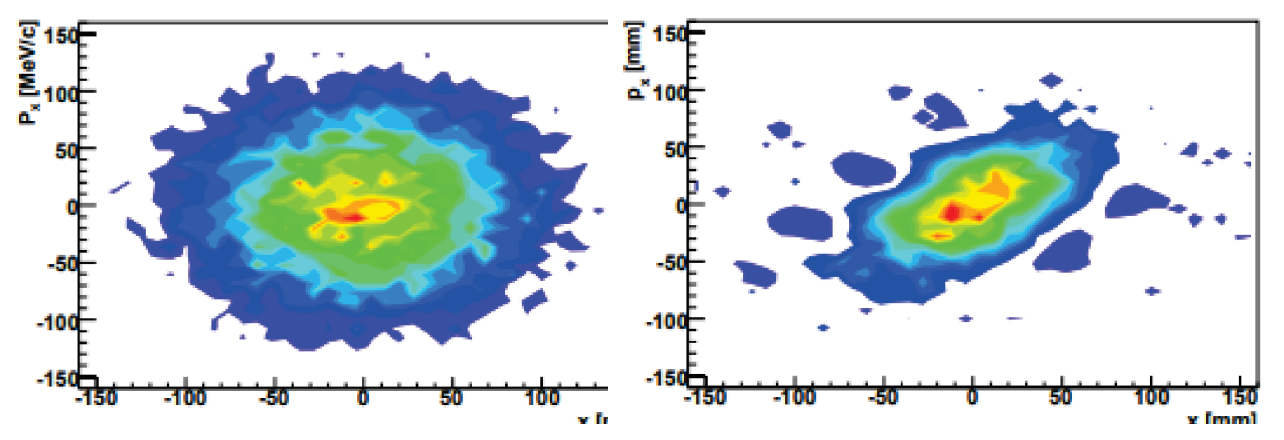

Figure 3: Illustration of the method used to create a dispersive beam in MICE. Left: initial multivariate gaussian. Right: created dispersive beam, after the muon rewighting procedure.

Choosing the correct weights to select for a sufficiently dispersive beam from an initial MICE distributed beam and tracking that beam through the cooling channel should allow for the demonstration of emittance exchange.

Preliminary Monte Carlo simulation results using G4beamline [14] of a muon beam travelling from the upstream reference plane through the wedge to the downstream reference plane are shown in figure 4 (for more details see reference [15]).
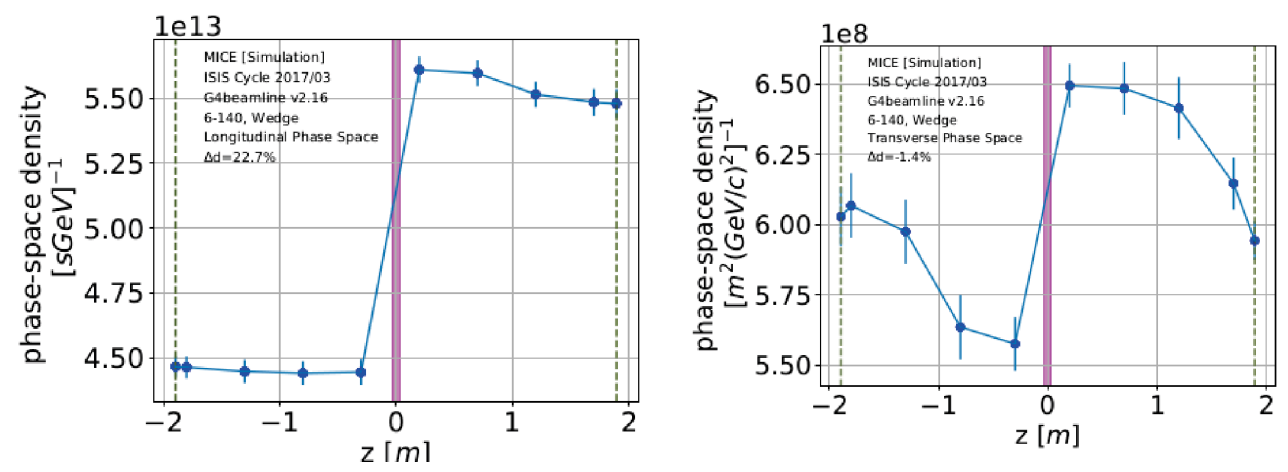

Figure 4: Left: increase in the longitudinal core phase space density across the wedge absorber (MC simulation). Right: change in transverse core phase space density in the same conditions.

The beam input emittance is $6 \mathrm{~mm}$ and the reference momentum $140 \mathrm{MeV} / \mathrm{c}$. About 88000 simulated muons were tracked. Currents in both the spectrometer solenoids and the absorber focus coils were set in the simulation to the ones used in MICE data taking.

An increase in the longitudinal component and a small decrease in the transverse component of the core phase-space density are seen in figure 4 , going from the upstream tracker reference plane $(z \sim-1.8 \mathrm{~m})$ to the downstream reference plane $(z \sim 1.8 \mathrm{~m})$.

Analysis of the collected data is in progress and will be available soon. 


\section{Conclusions}

The measurement of emittance exchange in MICE is in progress and has been shown feasible with dedicated simulations. The needed dispersive beam will be selected by a weighting procedure applied on single particle muon measurements. A sample of about 14 million triggers has been collected and is presently under study.

\section{References}

[1] A.N. Skrinsky and V.V. Parkhomchuk, Sov. J. Part. Nucl. 12 (1981) 223-247; D. Neuffer, Part. Accel. 14 (1988) 75-90.

[2] M.A. Palmer, proceedings COOL13, FERMILAB-CONF-13-253-APC; D. Neuffer et al., JINST 12 (2017) T07003.

[3] G. Gregoire et al., proposal to RAL, 2003.

[4] M. Bogomilov et al. [MICE Collaboration] JINST 7 (2012) P05009

[5] D. Adams et al. [MICE Collaboration] Eur. Phys. J. C73 (2013) 2582.

[6] R. Bertoni et al., Nucl. Instr. and Meth. A615 (2010) 14, arXiv:001.4426; R. Bertoni et al., MICE note 254 (2010).

[7] D. Adams et al. [MICE Collaboration] JINST 10 (2015) no 12, P12012

[8] M. Ellis et al., Nucl. Instr. and Meth. A655 (2011) 136.

[9] V.Blackmore et al. [MICE Collaboration] Eur. Phys. J. C79 (2019) no 3257

[10] M. Bogomilov et al. Phys. ReV. Accel Beams 20 (2017) no 06, 063501.

[11] S.Ozaki et al., BNL-52623, June 2001 ; M.M. Alsharo'a et al.,Phys. Rev. ST. Accel. Beams 6, 081001 (2003).

[12] M. Bogomilov et al. [MICE Collaboration], arXiv:1907.08562 [physics.acc-ph], submitted to Nature.

[13] C.T. Rogers, EPAC08 conference, Genova, Italy, paper TUPC088, 1260-1262.

[14] T.Roberts, proceedings 2007 IPAC Conference, DOI:10.1109/IPAC.2007.444.0461

[15] A. Tanaz Mohayai, FERMILAB-THESIS-2018-31, PhD thesis IIT 2018. 